\title{
Preliminary Trajectory Design for Cis-Lunar Libration Point Mission
}

\author{
Salman Ali Thepdawala ${ }^{1}$ \\ ${ }^{1}$ Skolkovo Institute of Science \& Technology, Space Centre \\ Bolshoy Boulevard 30, bld. 1, Moscow, Russia - 121205 \\ SalmanAli.Thepdawala@skoltech.ru
}

\begin{abstract}
We consider a mission design study in an Earth-Moon Circular Restricted Three-Body Problem (CRTBP) undertaken by Skoltech. We are to design a preliminary cis-lunar trajectory of a spacecraft from geostationary Earth orbit (GEO) to the L2 Halo orbit for the purpose of Lunar far side observation. The circular restricted three-body model is used and the libration point dynamics are exploited for the purpose of mission design. In the absence of any systematic approach for libration point missions, we use the existing mathematical tools for the purpose of mission design. In particular, this study makes use of dynamical systems theory and the invariant manifolds technique. This paper gives a brief overview of the steps involved in a preliminary mission design approach in a low-fidelity model. It utilizes a differential evolution optimization algorithm to optimize the transfer in terms of the required change in velocity from GEO to the stable manifold. It also discusses future work to refine the preliminary results applicable to high-fidelity models.
\end{abstract}

Keywords: Invariant Manifolds; Halo Orbit; GEO; Trajectory Optimization; Circular Restricted Three Body Problem (CRTBP); Cislunar Trajectory; Lunar far side; Trade-off

\section{Introduction}

The study of Circular Restricted Three-Body Problem (CRTBP) with Libration Point Orbits (LPO) for space trajectory design has been of great interest. Making use of the properties of CRTBP and relative equilibrium points, also known as Lagrange or Libration points, several studies on the problem of designing low-cost trajectories exists [1]. As the Lagrange points are at rest with respect to the primary bodies, the periodic orbits about these points offer an opportunity to implement innovative space missions $[2,3,4,5]$. There have been many missions in regions near the Sun-Earth Lagrange points, but very few near the Earth-Moon Libration point. It was not until the ARTEMIS mission successfully inserted a probe into the Earth-Moon L2 orbit in August 2010 [6] that more attention was paid towards the Lagrange points in cislunar space. Given ARTEMIS's success and the potential advantages of the Lagrange points, such as communications and scientific activities, the interest in multi-body trajectories and the number of missions in cis-lunar space has been ever increasing.

A particular class of LPOs, halo orbits, offers various new-concept space missions because of their unique features. In particular, following the ideas of Farquhar [2], a satellite can be placed at the L2 libration point to have a real-time communication link between the far side of the Moon and the Earth [7]. By placing another single relay satellite at the cislunar libration point L1, we can cover most of the lunar surface, and a point-to-point communication network could be established as well [8]. Other applications include the possibility to set a permanent space station at L2 by Heppenheimer [9], and on free-fall trajectories between the Libration points and the primaries by Broucke [10] and Prado [11].

In order to design the trajectories leading to halo orbits, they have been extensively studied, and their dynamical properties have been exploited. Further improvements have been made in recent years by treating the problem under the perspective of dynamical systems theory [12]. It played an essential role in the trajectory design of the Genesis mission [13]. The cheapest and well-established method for Earth-to-halo transfers is to place the spacecraft on the stable manifold associated with the target halo orbit $[12,14,15]$. Once on this manifold, the spacecraft will reach its target halo orbit under the influence of the dynamical system.

In the Earth-Moon system, the stable manifold associated with the halo orbits does not approach Earth. It does not allow a direct transfer from a low earth orbit with a single impulse maneuver as compared to the Sun-Earth system. Therefore, to overcome this, an indirect method uses Lambert's problem in a three-body context and represents a Two- 
Point Boundary Value Problem (TPBVP). However, Lambert's problem, in three body contexts, no longer has analytical solutions. Therefore, Lambert's problem can be solved only through numerical techniques. D'Amario and Edelbaum [16] and $\mathrm{Pu}$ and Edelbaum [17] studied the optimal Lambert's arcs in the three and four-body model.

This paper is organized into five main sections. The problem statement is presented in Section 2. The circular restricted three-body problem, which is the essence of this entire study, and the subsequent study on halo orbits and invariant manifolds are briefly covered in Section 3. Section 4 talks about the procedure followed to generate the desired results. The results are then displayed and discussed in Section 5. Finally, the paper is concluded in Section 6 with comments on the importance of simplicity of the procedure for the preliminary mission design phase and discussion on future work for a detailed mission design study in a high-fidelity model.

\section{Problem Statement}

We consider a mission design of a spacecraft traveling from a geostationary Earth orbit (GEO) to the Earth-Moon L2 Halo orbit based on a piggyback launch opportunity available. The preliminary mission design is carried out such that the spacecraft's trajectory in GEO is connected to the stable manifold trajectory associated with the L2 Halo orbit via Lambert's arc utilizing the Two-Point Boundary Value Problem (TPBVP). It then follows a predefined low energy trajectory within Circular Restricted Three-Body Problem (CRTBP) dynamics from the Stable Manifold Injection Point (SMIP) to the L2 Halo orbit. The focus is to get a low enough value of required change in velocity for the entire mission, which is comparable with the literature and can serve as a foundation for preliminary analysis.

\section{Circular Restricted Three Body Problem}

A limited case of the N-Body problem is presented here using the CRTBP. In this model, we consider Earth and Moon as the two primaries. Due to its low mass, the third body (spacecraft) is considered to have no effect on the motion of the primaries. The two primaries are assumed to form an isolated two-body system, where each moves in a circular orbit around their common barycentre. The origin of the coordinate frame is fixed at the barycentre and the entire system is viewed as a rotating frame following the rotation of the primaries. For simplicity, we will use non-dimensional units such that the distance unit (DU), time unit (TU), velocity unit (VU), mass unit (MU), and mass ratio ( $\mu$ ) are as given in Table 1. Subsequently, the primaries are located at $x=(1-\mu)$ and $x=-\mu$. Hence, the equations of motion for the spacecraft in CRTBP can be written as [18]:

$$
\ddot{x}-2 \dot{y}=\frac{\partial U}{\partial x}, \quad \ddot{y}+2 \dot{x}=\frac{\partial U}{\partial y}, \quad \ddot{z}=\frac{\partial U}{\partial z},
$$

Where $x, y, z$ are the third body position vector components with respect to the reference frame, and their first and second time derivatives represent velocity and acceleration, respectively, $U$ is the effective potential and is given by the following expression:

$$
\begin{gathered}
U=\frac{1}{2}\left(x^{2}+y^{2}\right)+\frac{\mu-1}{r_{13}}+\frac{\mu}{r_{23}}, \\
r_{13}=\sqrt{(x+\mu)^{2}+y^{2}+z^{2}}, \\
r_{23}=\sqrt{(x-1+\mu)^{2}+y^{2}+z^{2}},
\end{gathered}
$$

where, $r_{13}$ and $r_{23}$ represent distance of the spacecraft from the first and second primaries, respectively.

Table 1: Characteristic Quantities

\begin{tabular}{|c|c|c|c|}
\hline Name & Constants & Values & Units \\
\hline Mass Ratio & $\mu$ & 0.01215780332478703 & NonDim \\
\hline Unit Distance & DU & 384747.962856037 & $\mathrm{~km}$ \\
\hline Unit Mass & MU & $6.04550000 \cdot 10^{24}$ & $\mathrm{~kg}$ \\
\hline Unit Time & TU & 375727.551633535 & $\mathrm{~s}$ \\
\hline Unit Velocity & VU & 1.0240078513893494 & $\mathrm{~km} / \mathrm{s}$ \\
\hline
\end{tabular}




\subsection{Halo Orbit}

The model under the CRTBP dynamics allows the existence of a variety of orbits around Libration points [19]. In this study, we have considered a periodic halo orbit with an out-of-plane altitude of $8,000 \mathrm{~km}$, which satisfies the design requirement of Skoltech's tentative mission plan. The procedure for computation of relevant Halo orbits [12] using Richardson's third-order approximation [20] and differential correction using the Newton-Raphson method [21] are well documented in the literature. The initial state of the considered Halo orbit and its time period is listed in Table 2, with plots shown in Figure 1.

Table 2: Initial State: Halo Orbit

\begin{tabular}{|c|c|c|c|c|c|c|}
\hline $\mathbf{x}(\mathbf{D U})$ & $\mathbf{y}(\mathbf{D U})$ & $\mathbf{z}(\mathbf{D U})$ & $v_{x}(\mathbf{V U})$ & $v_{y}(\mathrm{VU})$ & $v_{z}(\mathrm{VU})$ & Time Period $(\mathrm{TU})$ \\
\hline 1.12040641 & 0.00 & $1.03488330 \mathrm{e}-03$ & 0.00 & $1.76059111 \mathrm{e}-01$ & 0.00 & 1.70777104 \\
\hline
\end{tabular}

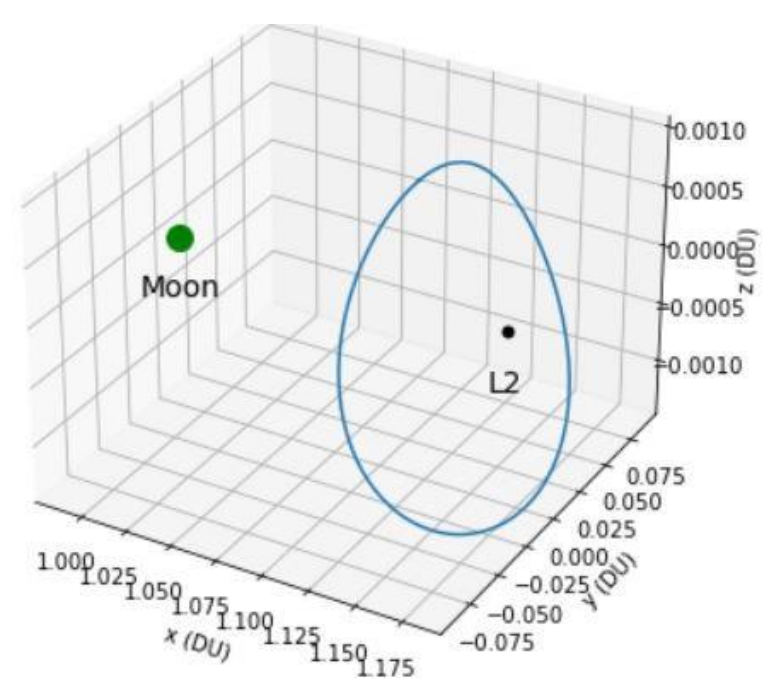

(a) 3D View

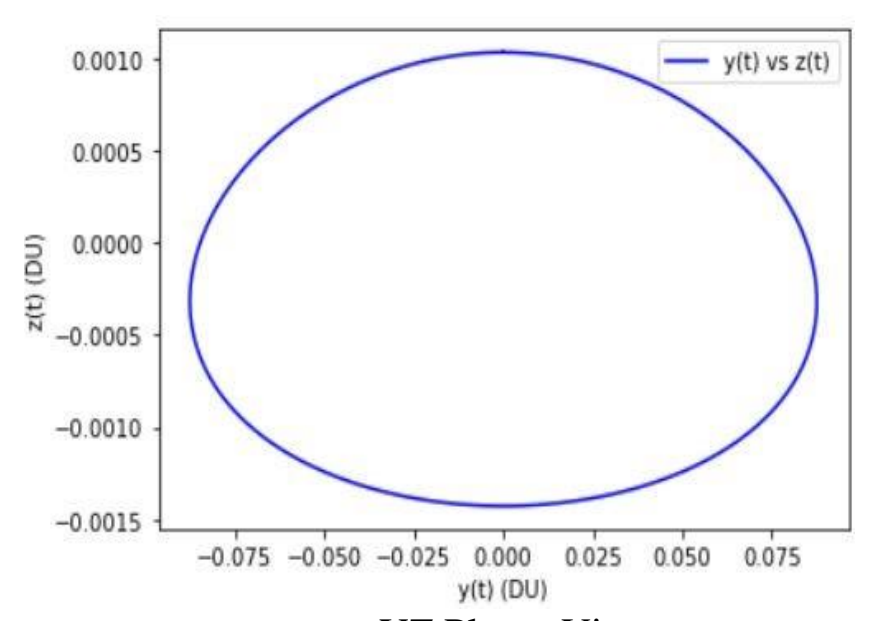

(b) YZ Planar View

Figure 1: L2 Halo Orbit in Earth-Moon CRTBP

\subsection{Computation of Invariant Manifolds}

Libration point, L2, being dynamically unstable, can be modelled as a saddle point. It gives rise to the stable and unstable manifolds associated with the Halo orbits at L2. More details on computation and analysis of invariant manifolds can be found in [22]. The Monodromy matrix, which exists for periodic orbits and is computed by propagating state transition matrix for one entire orbit, plays a vital role in the computation of invariant manifolds associated with any specific Halo orbit. Eigenvalues $\left(\lambda_{i}\right)$ of the monodromy matrix will always follow the following formulation:

$$
\lambda_{1}>1, \quad \lambda_{2}=\frac{1}{\lambda_{1}}, \quad \lambda_{3}=\lambda_{4}=1, \quad \lambda_{5}=\bar{\lambda}_{6}, \quad\left\|\lambda_{5}\right\|=1,
$$

Since halo orbits are periodic, two eigenvalues will always equal one, two will be complex conjugates, and two will be real. The pair of complex conjugates are related to quasi-periodic orbits around the libration point and hence fall outside the scope of this study. The pair of real eigenvalues $\left(\lambda_{1}, \lambda_{2}\right)$ are the ones associated with the unstable and stable manifold respectively and are exploited for the purpose of mission design.

\section{Solution Methodology and Genetic Algorithms}

Once we have the halo orbit (Table 2), we then find the local approximations to the stable manifold of the said halo orbit from the eigenvector (corresponding to the stable eigenvalue) of the monodromy matrix. This local approximation of the stable manifold is then integrated backward in time using the CRTBP nonlinear Eq (1). Given the formulation, we generate approximately hundred and one time-continuous, stable trajectories on the manifold shown in Figures 2 and 3. Each point on a single trajectory can be linked with the spacecraft's position in GEO through Lambert's arc for a given time of flight, resulting in an infinite number of options to choose from. This formulation of the problem represents the 
well-known Lambert's problem, and in this case under the influence of three-body environment, is called Lambert's three body problem, explained in [23]. However, evaluating solutions at each point in every trajectory requires excessive computational time. Since we are still in the preliminary design phase, we choose a point nearest to Earth for each trajectory and evaluate the solution for the selected points.

For the problem at hand, the main variables affecting the required change in velocity are the flight time for Lambert's arc and the position of spacecraft in GEO, true anomaly. A slight change in either can have a high impact on the final solution owing to the non-linearity. As we aim to minimize the required change in velocity, we are presented with an optimization problem based on two variables. Therefore, we decided to go with a genetic algorithm, in particular differential evolution, for its ability to find the global minimum of a multivariate function. We then run the standard SciPy differential evolution optimizer with the variable bounds given in Table 3. Bounds for true anomaly are such that every possible position in GEO is explored for the optimal solution. However, bounds for flight time are based on the fact that since the SMIP is the closest to Earth, and as observed during the development of the algorithm, the optimal solution should lie within 50 hours of flight time.

Table 3: Variable Bounds for Differential Evolution

\begin{tabular}{|c|c|c|}
\hline Parameter & Bounds & Units \\
\hline Time of Flight (for Lambert's Arc) & {$[1-50]$} & Hours \\
\hline True Anomaly (Position of Spacecraft in GEO) & {$[0-359]$} & Degrees \\
\hline
\end{tabular}

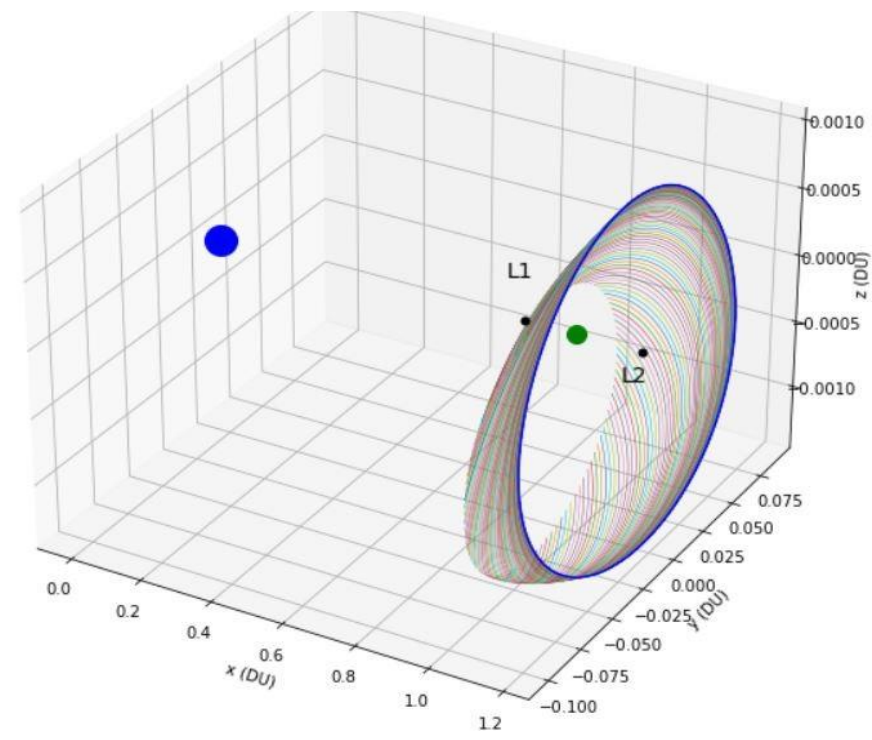

(a) 3D View of 101 stable trajectories on a manifold at the onset of propagation

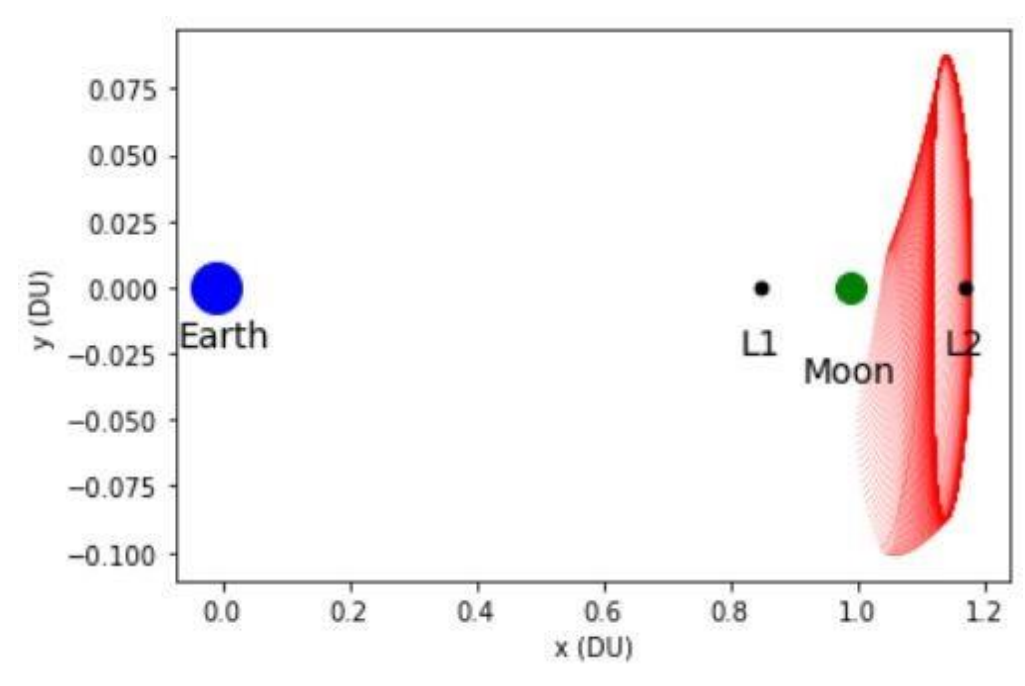

(b) XY Planar View of 101 stable trajectories on a manifold at the onset of propagation

Figure 2: Stable Manifold Trajectories for the stated Halo Orbit 


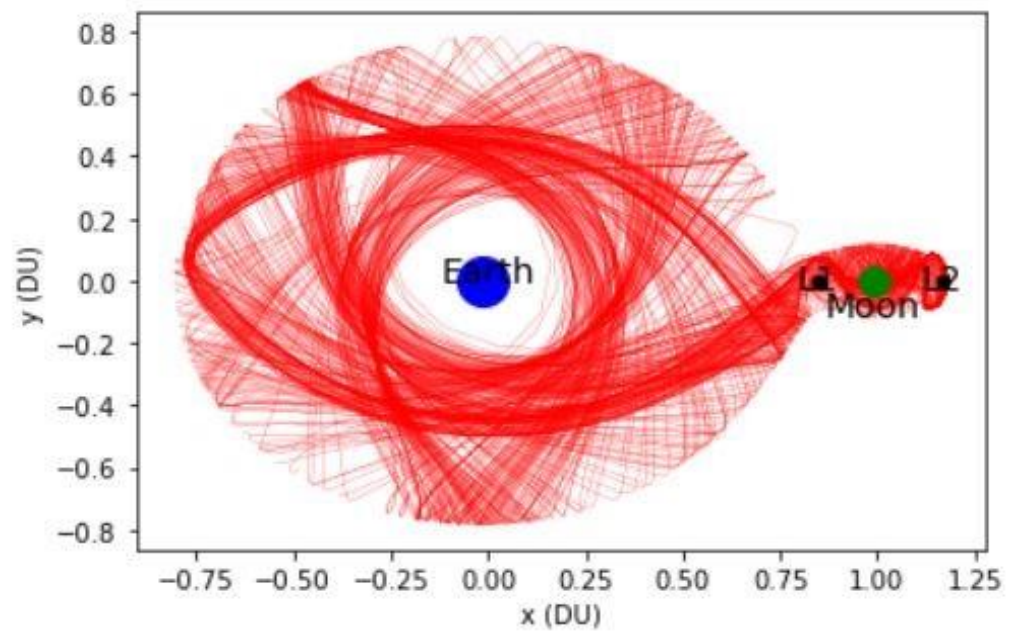

\section{Results}

Figure 3: 101 Stable trajectories on a manifold propagated for 100 days

Evaluating solutions for the closest point to Earth on each stable manifold yielded hundred and one different results for a total change in velocity and the corresponding total time of flight for each trajectory. Table 4 shows the optimized value for the required total change in velocity and its corresponding total flight time. For comparison, it also shows the minimum solution for a total time of flight among the hundred and one points evaluated. It can be easily observed that the optimized velocity solution takes approximately twice as much time as compared to the time optimized solution. Looking at these two selected cases and ninety-nine other solutions, one can easily make a trade-off analysis per their mission design constraints. It is important to note that the optimized value obtained in this study is in proximity with the values present in the literature [24].

Table 4: Values for minimized required change in velocity and minimized time of flight solutions

\begin{tabular}{|c|c|c|c|}
\hline No. & Minimized Solution for: & Change in Velocity $\mathbf{( k m} / \mathbf{s})$ & Time of Flight (days) \\
\hline 1. & Required Change in Velocity & 1.201 & 95.6 \\
\hline 2. & Total Time of Flight & 1.466 & 42.0 \\
\hline
\end{tabular}

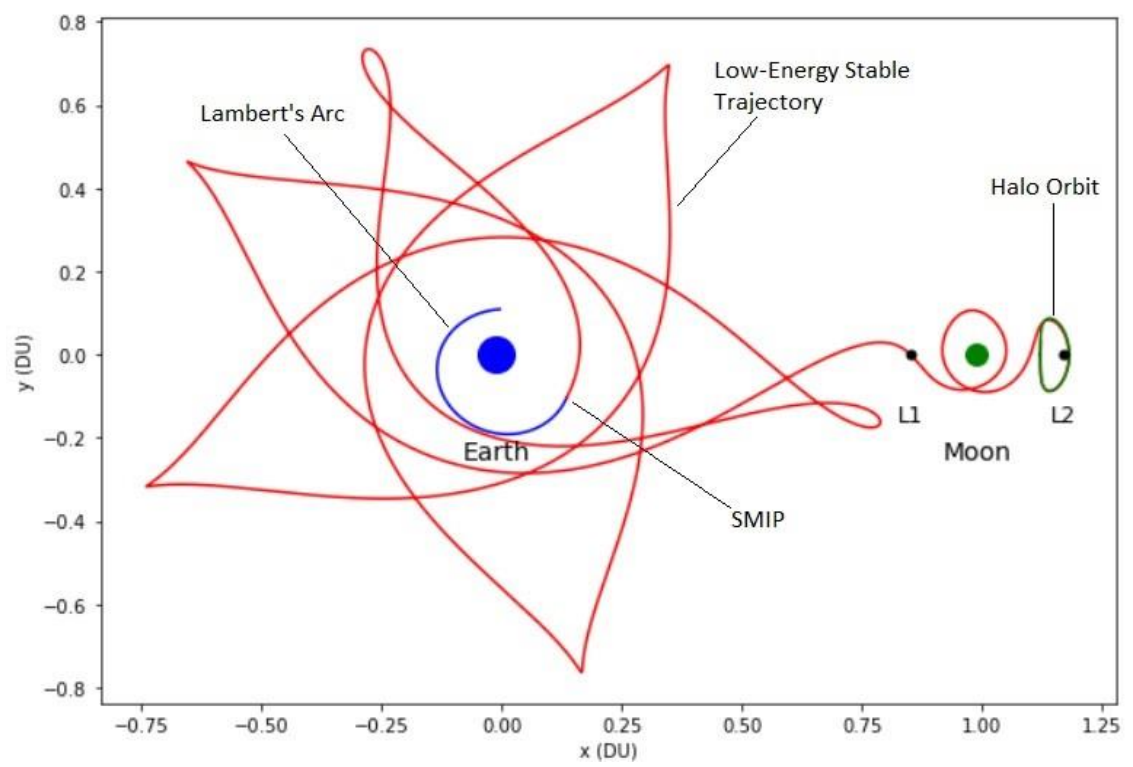

Figure 4: Graph showing optimized low-cost trajectory transfer. First row solution in Table 4 
Figure 4 demonstrates the optimized velocity solution graphically. Trajectory in blue corresponds to Lambert's arc. Red corresponds to the stable manifold trajectory. The point where they meet is the Stable Manifold Insertion Point (SMIP), and green corresponds to the halo orbit, as shown in Figure 1.

\section{Conclusion}

The preliminary mission design task, as explained above, leverages the multi-body dynamics of the cis-lunar space for computationally inexpensive low-energy trajectories from GEO to L2 Halo orbit. Results achieved in this work can be best counted as preliminary but offer valuable insight into the process of mission design and a fair estimate of the total change in velocity required for the mission design. Furthermore, being reasonably straightforward, the outlined procedure does not involve any time-consuming complex processes requiring high computational resources and can yield decent estimates in a short time for the preliminary phase, as discussed in Section 5.

The future work entails applying optimal control theory to the CRTBP mission design problem. It will allow us to optimize the objective function regardless of any singular point on a specific trajectory, yielding a true minimum. Once this is done, in order to be mission-ready, this entire problem formulation is to be fed into a high-fidelity model. For this purpose, we can avail the open-source SPICE kernels available online [25].

\section{References}

[1] G Mingotti, Francesco Topputo, and Franco Bernelli-Zazzera. Combined optimal low-thrust and stable-manifold trajectories to the earth-moon halo orbits. In AIP Conference Proceedings, volume 886, pages 100-112. American Institute of Physics, 2007.

[2] ROBERT W FARQUHAR. Lunar communications with libration-point satellites. Journal of Spacecraft and Rockets, 4(10):1383-1384, 1967.

[3] Robert W Farquhar. Future missions for libration-point satellites. Astronautics Aeronautics, 7:52-56, 1969.

[4] GL Condon and DJ Pearson. The role of humans in libration point missions with specific application to an earth-moon libration point gateway station. Advances in the Astronautical Sciences, 2002.

[5] Kevin E Post, Edward Belbruno, and Francesco Topputo. Efficient cis-lunar trajectories. In Global Space Exploration Conference, pages 1-19. Citeseer, 2012.

[6] David C Folta, Mark Woodard, Kathleen Howell, Chris Patterson, and Wayne Schlei. Applications of multi-body dynamical environments: the artemis transfer trajectory design. Acta Astronautica, 73:237-249, 2012.

[7] LiHua ZHANG, Liang XIONG, Ji SUN, Shan GAO, XiaoLei WANG, and AiBing ZHANG. Technical characteristics of the relay communication satellite "queqiao" for chang'e-4 lunar farside exploration mission. SCIENTIA SINICA Technologica, 49(2):138-146, 2019.

[8] W Farquhar. A halo-orbit lunar station. 1972.

[9] TA Heppenheimer. Steps toward space colonization-colony location and transfer trajectories. Journal of Spacecraft and rockets, 15(5):305-312, 1978.

[10] R Broucke. Traveling between the lagrange points and the moon. Journal of Guidance and Control, 2(4):257-263, 1979.

[11] Antonio FBA Prado. Traveling between the lagrangian points and the earth. Acta Astronautica, 39(7):483-486, 1996.

[12] Kathleen C Howell, Brian T Barden, and MARTIN W Lo. Application of dynamical systems theory to trajectory design for a libration point mission. The Journal of the Astronautical Sciences, 45(2):161-178, 1997.

[13] Wang Sang Koon, Martin W Lo, Jerrold E Marsden, and Shane D Ross. The genesis trajectory and heteroclinic connections. In AAS/AIAA Astrodynamics Specialist Conference, pages 99-451. Citeseer, 1999.

[14] Gerard Gómez, A Jorba, Jt Masdemont, and C Simó. Study of the transfer from the earth to a halo orbit around the equilibrium point 11. Celestial Mechanics and Dynamical Astronomy, 56(4):541-562, 1993.

[15] Anna Zanzottera, Giorgio Mingotti, Roberto Castelli, and Michael Dellnitz. Intersecting invariant manifolds in spatial restricted three-body problems: design and optimization of earth-to-halo transfers in the sun-earth-moon scenario. Communications in Nonlinear Science and Numerical Simulation, 17(2):832-843, 2012. 
[16] LOUIS A D'AMARIO and Theodore N Edelbaum. Minimum impulse three-body trajectories. AIAA Journal, 12(4):455-462, 1974.

[17] CL Pu and TN Edelbaum. Four-body trajectory optimization. AIAA Journal, 13(3):333-336, 1975.

[18] Sandeep K Singh, Brian D Anderson, Ehsan Taheri, and John L Junkins. Exploiting manifolds of 11 halo orbits for end-to-end earth-moon low-thrust trajectory design. Acta Astronautica, 2021.

[19] Kathleen C Howell. Families of orbits in the vicinity of the collinear libration points. The Journal of the astronautical sciences, 49(1):107-125, 2001.

[20] David L Richardson. Analytic construction of periodic orbits about the collinear points. Celestial mechanics, 22(3):241-253, 1980.

[21] Kathleen Connor Howell. Three-dimensional, periodic, 'halo'orbits. Celestial mechanics, 32(1):53-71, 1984.

[22] Wang Sang Koon, Martin W Lo, Jerrold E Marsden, and Shane D Ross. Dynamical systems, the three-body problem and space mission design. In Equadiff 99: (In 2 Volumes), pages 1167-1181. World Scientific, 2000.

[23] Sara Manglativi, Antonino Campolo, Bastien Le Bihan, and Stéphanie Lizy-Destrez. Safety analysis for near rectilinear orbit close approach rendezvous in the circular restricted three-body problem. 2017.

[24] Franco Bernelli Zazzera, Francesco Topputo, and Mauro Massari. Assessment of mission design including utilization of libration points and weak stability boundaries. 2004.

[25] Charles H Acton Jr. Ancillary data services of nasa's navigation and ancillary information facility. Planetary and Space Science, 44(1):65-70, 1996. 